\title{
The effect of a prevention procedure modified by evidence- based nursing on the incidence of lower extremity deep venous thrombosis after stroke
}

\author{
Yanxia Huang ${ }^{\# 1}$, Huiping Peng ${ }^{\# 1}$, Qiaogen Chen ${ }^{\# 1}$, Xin Zhang ${ }^{1}$, Xiujun Fan ${ }^{1}$, Lilan Xiao ${ }^{1}$, Hongmei Tao ${ }^{\ddagger 2}$ and Lei Zhang $^{*}$ \\ ${ }^{1}$ Department of Neurology, The Fifth Affiliated Hospital of Sun Yat-sen University, No 52 Meihuadong Road, Zhuhai, Guangdong, China \\ ${ }^{2}$ Department of Nurse, The Fifth Affiliated Hospital of Sun Yat-sen University, No 52 Meihuadong Road, Zhuhai, Guangdong, China \\ \#Authors contributed equally to the manuscript.
}

\begin{abstract}
Objective: To investigate the effect of deep venous thrombosis (DVT) prevention procedure on the incidence of lower extremity DVT after stroke.

Methods: The patients with stroke admitted in the Department of Neurology of The Fifth Affiliated Hospital of Sun Yat-sen University in 2015 were enrolled as the control group, and those admitted in 2016 were enrolled as the intervention group. The patients in the control group received routine preventive measures of lower extremity DVT. Based on the evidence-based medicine, doctors and nurses discussed and formulated a new procedure of lower extremity DVT prevention for patients with stroke. Based on the new procedure, the patients in the intervention group were assessed for the risk of DVT, and only the stroke patients with high risk of DVT received individualized preventive measures.
\end{abstract}

Results: The incidence of lower extremity DVT was significantly reduced in patients with stroke in 2016 comparing with that in 2015.

Conclusions: The new DVT prevention procedure based on evidence-based nursing can effectively prevent lower extremity DVT in patients with stroke.

\section{Introduction}

Deep venous thrombosis (DVT) and its sequel pulmonary embolism (PE) are important causes of illness and death after stroke [1]. In addition, about one-third of patients with symptomatic DVT develop the post-thrombotic syndrome, which is often disabling and is associated with substantial costs to patients and to society [2]. Patients who are immobile, with significant weakness of the limbs and with a prior history of DVT are at greatest risk of DVT development [3].

Although there are several options for thromboprophylaxis, many of these patients received no or inadequate thromboprophylaxis during their hospital stay [4]. In fact, because of inadequate thromboprophylaxis, pulmonary embolism is considered the principal cause of preventable death in hospitalized patients [5]. Therefore, the risk of DVT should be assessed in all patients with stroke on admission to hospital, and appropriate thromboprophylaxis should be prescribed. The aims of our study were to formulate a DVT prevention protocol for patients with stroke and investigate its efficacy.

\section{Methods}

\section{Ethics statement}

This research was approved by the ethics committee of The Fifth Affiliated Hospital of Sun Yat-sen University.

\section{Patients}

The study included all the patients who were diagnosed as having ischemic stroke for the first time and admitted in the Department of
Neurology of The Fifth Affiliated Hospital of Sun Yat-Sen University from January 2015 to December 2016.

\section{Diagnosis of ischemic stroke}

The diagnosis of ischemic stroke was based on the history of symptoms and their acute presentation, clinical examination and cerebral diffusion-weighted magnetic resonance imaging (DWI).

\section{Data collection and assessment}

The data on demographic characteristics, NIHSS scores, patterns of infarction distribution, risk factors of DVT, and occurrence of DVT were collected from medical documentation. The data of the patients with ischemic stroke admitted from January 2015 to December 2015 (phase 1) were retrospectively collected. During phase 1, only the patients with muscle strength of grade $0-3$ were taken into account for DVT prevention. The measures for DVT prevention included

Correspondence to: Lei Zhang, PhD, Department of Neurology, The Fifth Affiliated Hospital of Sun Yat-sen University, No 52 Meihuadong Road, Zhuhai, Guangdong, China, Tel: +86-7562528725, Fax: +86-7562528726, E-mail: zhang192@mail.sysu.edu.cn

Hongmei Tao, Master, Department of Nurse, The Fifth Affiliated Hospital of Sun Yat-sen University, No 52 Meihuadong Road, Zhuhai, Guangdong, China, Tel: +86-7562528725, Fax: +86-7562528726, E-mail: 13726209240@139.com

Key words: evidence-based nursing, prevention procedure, stroke, lower extremity deep venous thrombosis

Received: May 22, 2017; Accepted: June 07, 2017; Published: June 10, 2017 
intermittent pneumatic compression, passive activity, and avoidance of injections on the paralysed limbs. The causes of DVT among these patients were analyzed. A DVT prevention protocol for stroke patients was formulated by the doctors and nurses in the Department of Neurology, based on guidelines and literatures. The protocol was introduced from January 2016 to December 2016 (phase 2). The effect of the protocol on reducing DVT was assessed.

\section{The DVT prevention protocol for stroke patients}

From January 2016, the following protocol was implemented for the patients with ischemic stroke: 1) a Doppler ultrasound scan for four limbs was performed to detect preexistent DVT; 2) the doctors assessed the risk of DVT with Autar Scale, and gave medical orders of DVT prevention for the patients at moderate-high risk; 3) the nurses set up a nursing record for DVT detection. The contents of the nursing record included color, sensation, temperature, pulse strength, and circumference of the limbs, as well as Homans sign and Neuhof sign. If DVT was suspected, the doctors would be informed immediately and prescribed D-dimer test and Doppler ultrasound scan; 4) the nurses assessed the patients' muscle strength everyday and setting up individualized intervention: grade $0-2$, passive activity lasting 30 minutes on the bed, twice a day; grade $3-5$, active and passive activity; grade 0-3, intermittent pneumatic compression devices were applied for 15 minutes, twice a day; grade 4-5, patients were taught to do ankle pump; 5) venous protection: injections in the paralysed limbs, repeated injections, and injections with stimulating drugs should be avoided. Besides, drugs should be injected through the upper limbs instead of the lower limbs; 6) if necessary, the patients at high risk of DVT would receive anti-coagulation therapy; 7) when DVT was suspected, relative scales would be used to assess the probability of DVT; 8) when DVT was highly suspected, anti-coagulation therapy could be given before definite diagnosis was made, as long as there was no contraindication of anti-coagulation; 9) index of coagulation and signs of bleeding should be monitored during anti-coagulation therapy; 10) if necessary, patients would receive thrombolysis or thrombectomy; 11) dietary guide: low fat, low vitamin $\mathrm{K}$, and high fiber diet was recommended. Large amount of water intake was helpful to reduce blood viscosity; 12) dealt with constipation; 13) the doctors and nurses made dynamic assessment and discussion together during ward-round; 14) informing the nurses and doctors on the next shift about the DVT risk of all the stroke patients; 15) supervision of the DVT prevention measures by nursing group leaders and head nurse.

\section{Statistical analysis}

Statistical analysis was performed by SPSS version 19.0. P values of 0.05 were considered statistically significant. All quantitative data in this study are presented as mean \pm standard deviation (SD) or median \pm range. Quantitative data were processed using the $t$ test. Qualitative data were analyzed with the chi-square test.

\section{Results}

There were 348 and 419 patients with ischemic stroke admitted to our department in phase 1 and phase 2, respectively. The demographic characteristics, NIHSS scores, patterns of infarction distribution, and risk factors of DVT of these patients were summarized in Table 1. There was no significant difference in demographic characteristics, NIHSS scores, patterns of infarction distribution, and risk factors of DVT between the 2 groups. There were 6 cases of DVT in phase 1 , and 1 case of DVT in phase 2. The details of the DVT cases were summarized in Table 2. The incidence of DVT in phase 2 was significantly lower than that in phase $1(\mathrm{p}=0.025$, Table 1$)$.
Table 1. Comparison of ischemic stroke patients in phase 1 and phase 2.

\begin{tabular}{|c|c|c|c|}
\hline & Phase 1 & Phase 2 & p \\
\hline Age (years) & $61.2 \pm 13.38$ & $58.4 \pm 13.67$ & 0.649 \\
\hline Male (\%) & 64.7 & 66.8 & 0.541 \\
\hline Smoking (\%) & 41.66 & 46.77 & 0.166 \\
\hline Thrombocytosis (\%) & 15.51 & 21.24 & 0.500 \\
\hline BMI & $25.2 \pm 2.11$ & $26.0 \pm 2.06$ & 0.403 \\
\hline Lying in bed more than 3 days (\%) & 27.58 & 29.83 & 0.522 \\
\hline Deep vein chatheterization (\%) & 5.17 & 5.72 & 0.753 \\
\hline Hyperhomocysteinaemia (\%) & 36.20 & 41.76 & 0.120 \\
\hline Anticardiolipin antibody seroposivity (\%) & 2.81 & 2.91 & 0.890 \\
\hline Hypercoagulable states (\%) & 22.98 & 25.77 & 0.400 \\
\hline Severe systemic infection (\%) & 9.19 & 10.73 & 0.547 \\
\hline NIHSS & $6.1 \pm 4.5$ & $5.7 \pm 3.8$ & 0.674 \\
\hline Infarction distribution (\%) & & & \\
\hline Anterior circulation & 81.4 & 83.7 & 0.813 \\
\hline Posterior circulation & 16.9 & 15.0 & 0.704 \\
\hline Anterior and posterior circulation & 1.7 & 1.3 & 0.575 \\
\hline Incidence of DVT (\%) & 1.72 & 0.24 & 0.025 \\
\hline
\end{tabular}

\section{Discussion}

Ischemic stroke is a main cause of neurologic morbidity and mortality worldwide. Patients with ischemic stroke are at high risk of DVT due to various neurologic impairment and systemic factors. The consequences of DVT for the patient can be severe, including PE and even death. Thus, DVT prevention is very important for patients with ischemic stroke. However, many patients received no or inadequate thromboprophylaxis during admission. The aims of our study were to formulate an effective fall prevention protocol for the inpatient ischemic stroke patients.

It has been demonstrated that ischemic stroke patients with severe paralysis were more prone to DVT [6]. Therefore, we focused DVT prevention on patients with muscle strength of grade $0-3$. From the cases of DVT in phase 1 , we found only 2 patients were with severe paralysis. On one hand, it suggested that the DVT prevention methods we used in the past could effectively reduce the incidence of DVT in ischemic stroke patients with severe paralysis. On the other hand, it suggested that focusing on such patients was not enough. Many other factors could increase the incidence of DVT, including infection, anticardiolipin syndrome, deep vein catheterization. So, the patients with ischemic stroke should receive comprehensive assessment for risks of DVT. Autar Scale is used to evaluate the risk of DVT, and the Autar Scale score is positively correlated to the incidence of DVT. In this research, we used Autar Scale to recognize the risk factors of DVT and identify the patients at moderate-high risk of DVT. Individualized management was applied to the patients according to the DVT prevention protocol. Meanwhile, we emphasized dynamic assessment so that the plan could be adjusted in time when the patients' situation improved or deteriorated. Since the DVT prevention protocol for stroke patients was introduced, the incidence of DVT in patients with ischemic stroke was significantly decreased.

The limitation of our study was that a second Doppler ultrasound scan was performed only when the patient with symptoms of DVT, so it was likely that the patients with asymptomatic DVT would be missed. Besides, limited to the amount of the cases of DVT, we were not able to perform association analysis to find out the risk factors of DVT among stroke patients during hospitalization.

In conclusion, assessing DVT risk upon the ischemic stroke patient's admission plays an important part in the efforts for preventing DVT during hospitalization. The comprehensive DVT prevention 
Huang Y (2017) The effect of a prevention procedure modified by evidence-based nursing on the incidence of lower extremity deep venous thrombosis after stroke

Table 2. Cases of DVT among ischemic stroke patients.

\begin{tabular}{|c|c|c|c|c|c|c|c|}
\hline & & Age & Gender & $\begin{array}{l}\text { Muscle strength of } \\
\text { paralysed limbs }\end{array}$ & NIHSS & Infarction distribution & Risk factors of DVT \\
\hline \multirow{6}{*}{2015} & Case 1 & 46 & Male & 1 & 9 & Anterior circulation & Immobility, lying in bed more than 3 days \\
\hline & Case 2 & 66 & Male & 4 & 4 & Anterior circulation & Increased D-dimer \\
\hline & Case 3 & 57 & Male & 4 & 6 & Anterior circulation & Thrombocytosis, smoking \\
\hline & Case 4 & 76 & Female & 3 & 7 & Posterior circulation & Immobility caused by gouty arthritis \\
\hline & Case 5 & 72 & Female & 4 & 5 & Anterior circulation & Hyperhomocysteinaemia \\
\hline & Case 6 & 68 & Male & 4 & 3 & Anterior circulation & Severe pulmonary infection \\
\hline 2016 & Case 1 & 62 & Male & 4 & 4 & Anterior circulation & Memantine \\
\hline
\end{tabular}

protocol for ischemic stroke patients was effective in reducing the incidence of DVT.

\section{Conflicts of interest}

The authors declare that there are no conflicts of interest.

\section{References}

1. Geerts WH, Bergqvist D, Pineo GF, Heit JA, Samama CM, et al. (2008) Prevention of venous thromboembolism: American College of Chest Physicians Evidence-Based Clinical Practice Guidelines (8th Edition). Chest 133: 381S-453S. [Crossref]

2. Kearon C, O’Donnell M (2011) Graduated compression stockings to prevent venous thromboembolism in hospital: evidence from patients with acute stroke. Pol Arch Med Wewn 121: 40-43. [Crossref]
3. Dennis M, Sandercock P, Reid J, Graham C, Murray G, et al. (2011) Can clinical features distinguish between immobile patients with stroke at high and low risk of deep vein thrombosis? Statistical modelling based on the CLOTS trials cohorts. J Neurol Neurosurg Psychiatry 82: 1067-1073. [Crossref]

4. Wolberg AS, Rosendaal FR, Weitz JI, Jaffer IH, Agnelli G, et al. (2015) Venous thrombosis. Nat Rev Dis Primers 1: 15006. [Crossref]

5. Rathbun S (2009) Cardiology patient pages. The Surgeon General's call to action to prevent deep vein thrombosis and pulmonary embolism. Circulation 119: e480-2.

6. De Silva DA, Pey HB, Wong MC, Chang HM, Chen CP (2006) Deep vein thrombosis following ischemic stroke among Asians. Cerebrovasc Dis 22: 245-250. [Crossref]

Copyright: (C2017 Huang Y. This is an open-access article distributed under the terms of the Creative Commons Attribution License, which permits unrestricted use, distribution, and reproduction in any medium, provided the original author and source are credited. 